\title{
THE
}

\section{GDOP Bounds for GNSS Augmented with Range Information}

\author{
Peter F. Swaszek \\ University of Rhode Island, swaszek@uri.edu \\ Richard J. Hartnett \\ Kelly C. Seals
}

Follow this and additional works at: https://digitalcommons.uri.edu/ele_facpubs

\section{The University of Rhode Island Faculty have made this article openly available.} Please let us know how Open Access to this research benefits you.

\section{Terms of Use}

This article is made available under the terms and conditions applicable towards Open Access Policy Articles, as set forth in our Terms of Use.

\section{Citation/Publisher Attribution}

Swaszek, Peter F., Hartnett, Richard J., Seals, Kelly C., "GDOP Bounds for GNSS Augmented with Range Information," Proceedings of the 30th International Technical Meeting of The Satellite Division of the Institute of Navigation (ION GNSS+ 2017), Portland, Oregon, September 2017, pp. 4221-4235.

Available at: https://www.ion.org/publications/abstract.cfm?articlelD=15391

This Conference Proceeding is brought to you for free and open access by the Department of Electrical, Computer, and Biomedical Engineering at DigitalCommons@URI. It has been accepted for inclusion in Department of Electrical, Computer, and Biomedical Engineering Faculty Publications by an authorized administrator of DigitalCommons@URI.For more information, please contact digitalcommons-group@uri.edu. 


\title{
GDOP Bounds for GNSS Augmented with Range Information
}

\author{
Peter F. Swaszek, University of Rhode Island \\ Richard J. Hartnett, U.S. Coast Guard Academy \\ Kelly C. Seals, U.S. Coast Guard Academy
}

\section{BIOGRAPHIES}

Peter F. Swaszek is a Professor in the Department of Electrical, Computer, and Biomedical Engineering at the University of Rhode Island. His research interests are in statistical signal processing with a focus on digital communications and electronic navigation systems.

Richard J. Hartnett is a Professor of Electrical Engineering at the U.S. Coast Guard Academy, having retired from the USCG as a Captain in 2009. His research interests include efficient digital filtering methods, improved receiver signal processing techniques for electronic navigation systems, and autonomous vehicle design.

Kelly C. Seals is the Chair of the Electrical Engineering program at the U.S. Coast Guard Academy in New London, Connecticut. He is a Commander on active duty in the U.S. Coast Guard and received a PhD in Electrical and Computer Engineering from Worcester Polytechnic Institute.

\begin{abstract}
Code phase GNSS receivers convert the measured satellite pseudoranges into estimates of the position and clock offset of the receiver, typically via an iterative, linearized least squares method. Since the pseudoranges themselves are noisy, the resulting estimates of position and time are random variables. To describe the accuracy of this solution, it is common to describe it statistically via the error covariance matrix. Rather than considering the individual elements of this covariance matrix, users frequently reduce it to a scalar performance indicator; the most common of these is the Geometric Dilution of Precision (GDOP).

It is well known that the GDOP is a function of the satellite geometry; with only a few visible satellites in poor locations, the GDOP can become quite large. However, for a future with multiple, fully occupied GNSS constellations it is expected that receivers would select those satellites to track so as to achieve the best possible performance. Hence, an understanding of both how small the GDOP can be as a function of the number of satellites visible and the characteristics of the constellations that meet that bound are of value. Further, once identified, a receiver could exploit those constellation characteristics in selecting a subset of satellites.

Investigating the best possible GNSS satellite constellation with respect to the GDOP is not a new problem. Recently, these authors developed achievable lower bounds to the GDOP as a function of the number of satellites; the bounds were also extended to non-zero mask angle and to multiple GNSS constellations. Further, using actual GPS satellite ephemeris data, it was shown by example that good GDOP performance resulted from constellations similar to the "best" constellations resulting from the bounds.

This paper examines augmentation of the GNSS pseudoragnes with data from non-GNSS sensors; specifically, ranges. While integration of GNSS and non-GNSS sensors is not novel, the perspective in the paper is how such external sensors impact potential receiver performance (i.e. minimum GDOP) and what role they play in satellite selection. Specifically, tight lower bounds to GDOP when the GNSS is augmented by this additional measurement (barometric altimeter or a DME slant range) are presented; achievability of the bounds is also examined.
\end{abstract}




\section{Introduction}

Code phase GNSS receivers convert the measured satellite pseudoranges into estimates of the position and clock offset of the receiver. The typical implementation of the solution algorithm is an iterative, linearized least squares method. Assuming that pseudoranges from non-coplanar satellites are measured, the direction cosines matrix $\mathbf{G}$ is formed and used to solve an over-determined set of equations to solve for receiver position and time offset.

Since the pseudoranges themselves are noisy, the resulting estimates of position and time are random variables. To examine the accuracy of this solution it is common to describe it statistically via the error covariance matrix, equal to the inverse of $\mathbf{G}^{T} \mathbf{G}$ scaled by the User Range Error, URE [1]. Rather than considering the individual elements of this covariance matrix, users frequently reduce it to a scalar performance indicator. The most common of these is the Geometric Dilution of Precision (GDOP), equal to $\sqrt{\operatorname{trace}\left\{\left(\mathbf{G}^{T} \mathbf{G}\right)^{-1}\right\}}$; equivalently, this is the square root of the sum of the variances of the estimates without the URE scaling. Other possible measures of performance are the Position (PDOP), Horizontal (HDOP), Vertical (VDOP), and Time (TDOP) portions of GDOP.

It is known that the GDOP is a function of the satellites' geometry; with only a few visible satellites in poor locations, the GDOP can become quite large. However, for a future with multiple, fully occupied GNSS constellations it is expected that receivers would select those satellites to track so as to achieve the best possible performance; see, for example, $[2,3]$. Hence, we think that an understanding of both how small the GDOP can be as a function of the number of satellites visible and the characteristics of the constellations that meet that bound are of value. Further, once identified, a receiver could exploit those constellation characteristics in selecting a subset of satellites $[4,5]$.

Investigating the best possible GNSS satellite constellation with respect to GDOP is not a new problem. The case of $\mathrm{m}=4$ satellites, with reference to optimizing the tetrahedron formed by their locations, has been considered by multiple authors, see e.g. [6]. The best constellations of 4, 5 , and 6 satellites are described in [7]; the case of 5 satellites from two GNSS constellations is considered in [8]. A general lower bound for $m$ satellites from one constellation is known [4], but does not restrict the satellites elevations to be above the horizon or above any fixed mask angle and, hence, is not achievable in terrestrial applications. In a recent paper [9], these authors were able to improve on this result, developing an achievable lower bound to GDOP for terrestrial applications

$$
\mathrm{GDOP} \geq \sqrt{\frac{2 \sqrt{6}+7}{m}}=\frac{3.45}{\sqrt{m}}
$$

The constellations that achieve this bound consist of approximately $29 \%$ of the satellites at zenith and the remaining $71 \%$ "balanced" around the horizon (balance having a specific mathematical definition, see [9]). In that same work the bounds were extended to PDOP, to non-zero mask angle, and to multiple GNSS constellations. Further, using actual GPS satellite ephemeris data, in [5] we showed by example that good GDOP/PDOP performance resulted from constellations similar to the "best" constellations resulting from the bounds.

In this paper we examine augmentation of the GNSS measurements with data from non-GNSS sensors, specifically range data, under the assumption that the data is integrated with the GNSS pseudoranges in the position solution. While integration of GNSS and non-GNSS sensors is not novel, here we show (from an optimal GDOP perspective) how such an external sensor impacts potential receiver performance and what role it plays in satellite selection. Specifically, we extend our results from [9], providing tight lower bounds to the GDOP when the GNSS is augmented by a single such measurement and present satellite constellations that achieve these bounds.

Some notes on the presented development and results include:

- While we do assume that the individual satellite errors are statistically equivalent (unbiased with common variance), we assume that the additional range measurement is of different accuracy; hence, the appropriate performance metric is a weighted GDOP.

- With no external sensor augmentation, the minimum GDOP constellations for GNSS alone are a 29\%-71\% split of satellites at zenith and balanced satellites at the horizon, respectively. The results for GDOP with augmentation by a range sensor are similar; the constellations that achieve the bounds are still a mix of horizon and zenith (or off-zenith, see below) satellites, but with modifications to the ratio and balance conditions based upon the direction and accuracy of the additional measurement source. 
- In our minds the case of an altimeter is an important application of these results. Specifically, we examine the required quality of the altitude measurement for the sensor to have a significant impact on GDOP and how an altimeter alters our view of satellite selection.

- If the relative accuracy of the additional measurement is much better than that of the GNSS satellites, we observe that diminishing returns result. Specifically, if the external sensor is nearly perfect, it still cannot drive the performance of the entire system beyond a point.

\section{GDOP with Range Augmentation}

Adding a single range measurement to the solution, the direction cosines matrix for $m$ satellites in three dimensions using an East, North, and Up coordinate frame is

$$
\mathbf{G}=\left[\begin{array}{cccc}
e_{0} & n_{0} & u_{0} & 0 \\
e_{1} & n_{1} & u_{1} & 1 \\
e_{2} & n_{2} & u_{2} & 1 \\
\vdots & \vdots & & \\
e_{m} & n_{m} & u_{m} & 1
\end{array}\right]
$$

in which $\left(e_{0}, n_{0}, u_{0}\right)$ is the unit vector pointing toward the ranging source and $\left(e_{k}, n_{k}, u_{k}\right), k=1,2 \ldots m$, are the unit vectors pointing toward the $m$ satellites, all from the receiver's location. (For notational convenience we have listed the range sensor first in this formulation; additional ranges could result in the occurrence of multiple such rows.) Further, let $\boldsymbol{\Gamma}$ be the matrix of variances of these measurements

$$
\boldsymbol{\Gamma}=\operatorname{diag}(\sigma_{r}^{2}, \underbrace{\sigma^{2}, \ldots \sigma^{2}}_{m \text { terms }})=\sigma^{2} \operatorname{diag}(\frac{\sigma_{r}^{2}}{\sigma^{2}}, \underbrace{1, \ldots 1}_{m \text { terms }})
$$

with the ratio $\frac{\sigma_{r}^{2}}{\sigma^{2}}$ accounting for the different accuracies of the systems ( $\sigma$ and $\sigma_{r}$ being the standard deviations of the GNSS pseudoranges and the augmentation range, respectively). Ignoring the leading $\sigma^{2}$ term, the "weighted" geometric dilution of precision is defined as

$$
\text { WGDOP }=\sqrt{\operatorname{trace}\left\{\left(\mathbf{G}^{T} \boldsymbol{\Gamma}^{-1} \mathbf{G}\right)^{-1}\right\}}
$$

with

$$
\mathbf{G}^{T} \boldsymbol{\Gamma}^{-1} \mathbf{G}=\left[\begin{array}{cccc}
\frac{\sigma^{2}}{\sigma_{r}^{2}} e_{0}^{2}+\sum_{k=1}^{m} e_{k}^{2} & \frac{\sigma^{2}}{\sigma_{r}^{2}} e_{0} n_{0}+\sum_{k=1}^{m} e_{k} n_{k} & \frac{\sigma^{2}}{\sigma_{r}^{2}} e_{0} u_{0}+\sum_{k=1}^{m} e_{k} u_{k} & \sum_{k=1}^{m} e_{k} \\
\frac{\sigma^{2}}{\sigma_{r}^{2}} e_{0} n_{0}+\sum_{k=1}^{m} e_{k} n_{k} & \frac{\sigma^{2}}{\sigma_{r}^{2}} n_{0}^{2}+\sum_{k=1}^{m} n_{k}^{2} & \frac{\sigma^{2}}{\sigma_{r}^{2}} n_{0} u_{0}+\sum_{k=1}^{m} n_{k} u_{k} & \sum_{k=1}^{m} n_{k} \\
\frac{\sigma^{2}}{\sigma_{r}^{2}} e_{0} u_{0}+\sum_{k=1}^{m} e_{k} u_{k} & \frac{\sigma^{2}}{\sigma_{r}^{2}} n_{0} u_{0}+\sum_{k=1}^{m} n_{k} u_{k} & \frac{\sigma^{2}}{\sigma_{r}^{2}} u_{0}^{2}+\sum_{k=1}^{m} u_{k}^{2} & \sum_{k=1}^{m} u_{k} \\
\sum_{k=1}^{m} e_{k} & \sum_{k=1}^{m} n_{k} & \sum_{k=1}^{m} u_{k} & m
\end{array}\right]
$$

With this formulation a number of the DOP bound results in [9] generalize. Our use of color in this matrix will become obvious below. 


\section{A Brief Review of [9]}

Following [9] the GDOP under GNSS alone is determined by the elements of the symmetric matrix $\mathbf{G}^{T} \boldsymbol{\Gamma}^{-1} \mathbf{G}$ with $\sigma_{r} \rightarrow \infty$ to account for no range sensor

$$
\lim _{\sigma_{r} \rightarrow \infty} \mathbf{G}^{T} \boldsymbol{\Gamma}^{-1} \mathbf{G}=\left[\begin{array}{cccc}
\sum_{k=1}^{m} e_{k}^{2} & \sum_{k=1}^{m} e_{k} n_{k} & \sum_{k=1}^{m} e_{k} u_{k} & \sum_{k=1}^{m} e_{k} \\
\sum_{k=1}^{m} e_{k} n_{k} & \sum_{k=1}^{m} n_{k}^{2} & \sum_{k=1}^{m} n_{k} u_{k} & \sum_{k=1}^{m} n_{k} \\
\sum_{k=1}^{m} e_{k} u_{k} & \sum_{k=1}^{m} n_{k} u_{k} & \sum_{k=1}^{m} u_{k}^{2} & \sum_{k=1}^{m} u_{k} \\
\sum_{k=1}^{m} e_{k} & \sum_{k=1}^{m} n_{k} & \sum_{k=1}^{m} u_{k} & m
\end{array}\right]
$$

Finding the minimum of GDOP was a four step procedure:

1. Set the off-diagonal terms to zero. Strictly we cannot do this for all of these terms; however, setting the ones shown in red (all but the $\sum_{k} u_{k}$ since each $u_{k} \geq 0$ ) is desired. Hence, we want

$$
\sum_{k=1}^{m} e_{k} u_{k}=0 \quad \sum_{k=1}^{m} e_{k}=0 \quad \sum_{k=1}^{m} n_{k} u_{k}=0 \quad \sum_{k=1}^{m} n_{k}=0 \quad \sum_{k=1}^{m} e_{k} n_{k}=0
$$

Effectively, these are conditions that the satellite constellation exhibit symmetry in multiple directions. In [9] these conditions, along with next one below, are used to define a "balanced" satellite constellation. See [5] for a more detailed discussion of balance.

2. Set the first two diagonal terms (in blue) to be equal to one another

$$
\sum_{k=1}^{m} e_{k}^{2}=\sum_{k=1}^{m} n_{k}^{2}
$$

We note that it is possible to satisfy all 6 of these conditions for any $m$ greater than 3 [5].

3. The impact of the remaining off-diagonal term on WGDOP (in black) is minimized by forcing all of the satellites to be at either zenith or on the horizon (elevations $90^{\circ}$ and $0^{\circ}$, respectively); let $\beta$ represent the fraction of the $m$ satellites at zenith, then the GDOP bound reduces to

$$
\mathrm{GDOP} \geq \sqrt{\frac{1+5 \beta}{m \beta(1-\beta)}}
$$

4. Finally, standard calculus provides the optimum value for $\beta$ is $\frac{\sqrt{6}-1}{5} \approx 0.29$ which yields the GNSS-only bound mentioned above.

To develop a lower bound for WGDOP that includes the range measurement, we intend to follow this same optimization process as much as possible with the modified matrix $\mathbf{G}^{T} \boldsymbol{\Gamma}^{-1} \mathbf{G}$. Since the direction to this range complicates the situation, we first consider two simple, yet important examples before examining the general case. 


\section{A Range to Nadir/Zenith}

Imagine that the ranging source is directly below or above the receiver so that $e_{0}=n_{0}=0, u_{0}= \pm 1$; an altimeter meets this definition with $u_{0}=-1$. With this restriction the matrix is

$$
\mathbf{G}^{T} \boldsymbol{\Gamma}^{-1} \mathbf{G}=\left[\begin{array}{cccc}
\sum_{k=1}^{m} e_{k}^{2} & \sum_{k=1}^{m} e_{k} n_{k} & \sum_{k=1}^{m} e_{k} u_{k} & \sum_{k=1}^{m} e_{k} \\
\sum_{k=1}^{m} e_{k} n_{k} & \sum_{k=1}^{m} n_{k}^{2} & \sum_{k=1}^{m} n_{k} u_{k} & \sum_{k=1}^{m} n_{k} \\
\sum_{k=1}^{m} e_{k} u_{k} & \sum_{k=1}^{m} n_{k} u_{k} & \frac{\sigma^{2}}{\sigma_{r}^{2}}+\sum_{k=1}^{m} u_{k}^{2} & \sum_{k=1}^{m} u_{k} \\
\sum_{k=1}^{m} e_{k} & \sum_{k=1}^{m} n_{k} & \sum_{k=1}^{m} u_{k} & m
\end{array}\right]
$$

and the optimization is as follows:

1. Set the red, off-diagonal terms to zero

$$
\sum_{k=1}^{m} e_{k} n_{k}=0 \quad \sum_{k=1}^{m} e_{k} u_{k}=0 \quad \sum_{k=1}^{m} e_{k}=0 \quad \sum_{k=1}^{m} n_{k} u_{k}=0 \quad \sum_{k=1}^{m} n_{k}=0
$$

Constellations meeting these conditions exist for all $m \geq 4$ as described in [9].

2. Set the first two diagonal terms (in blue) equal to one another

$$
\sum_{k=1}^{m} e_{k}^{2}=\sum_{k=1}^{m} n_{k}^{2}
$$

This, too, can always be satisfied.

3. Restrict the satellites to zenith or horizon. Let $\beta$ represent the fraction (a total of $\beta m$ satellites) at zenith and define the quality ratio $\gamma$ by

$$
\gamma^{2}=\frac{\sigma^{2}}{m \sigma_{r}^{2}}
$$

( $\gamma$ is effectively the ratio of the GNSS-alone accuracy, $\sigma / \sqrt{m}$, and the range accuracy, $\sigma_{r}$ ), the bound becomes

$$
\text { WGDOP }\left.\right|_{z e n i t h} \geq \sqrt{\left(\frac{4}{1-\beta}+\frac{1+\beta+\gamma^{2}}{\beta(1-\beta)+\gamma^{2}}\right) \frac{1}{m}}
$$

4. Setting a derivative to zero, the necessary condition on $\beta$ is

$$
5 \beta^{4}+\left(2 \gamma^{2}-8\right) \beta^{3}-12 \gamma^{2} \beta^{2}+\left(10 \gamma^{2}+4\right) \beta+4 \gamma^{4}-1=0
$$

We show in Appendix A that this quartic polynomial has exactly one positive real root if $\gamma \leq \frac{1}{\sqrt{2}}$; otherwise it has no positive roots so we use $\beta=0$ to minimize the lower bound.

Figure 1 shows the results versus the sensor standard deviation ratio, $\gamma$ :

- The top subplot shows that fraction of satellites that should be at zenith, $\beta$. As expected, for vanishingly

small $\gamma$ this ratio approaches 29\%, the fraction found in [9] for GNSS alone. Also, once $\gamma^{2}$ exceeds one-half (indicated by the vertical black dotted line), the ranging sensor obviates the need for zenith satellites and all $m$ should be balanced about the horizon for minimum WGDOP. 

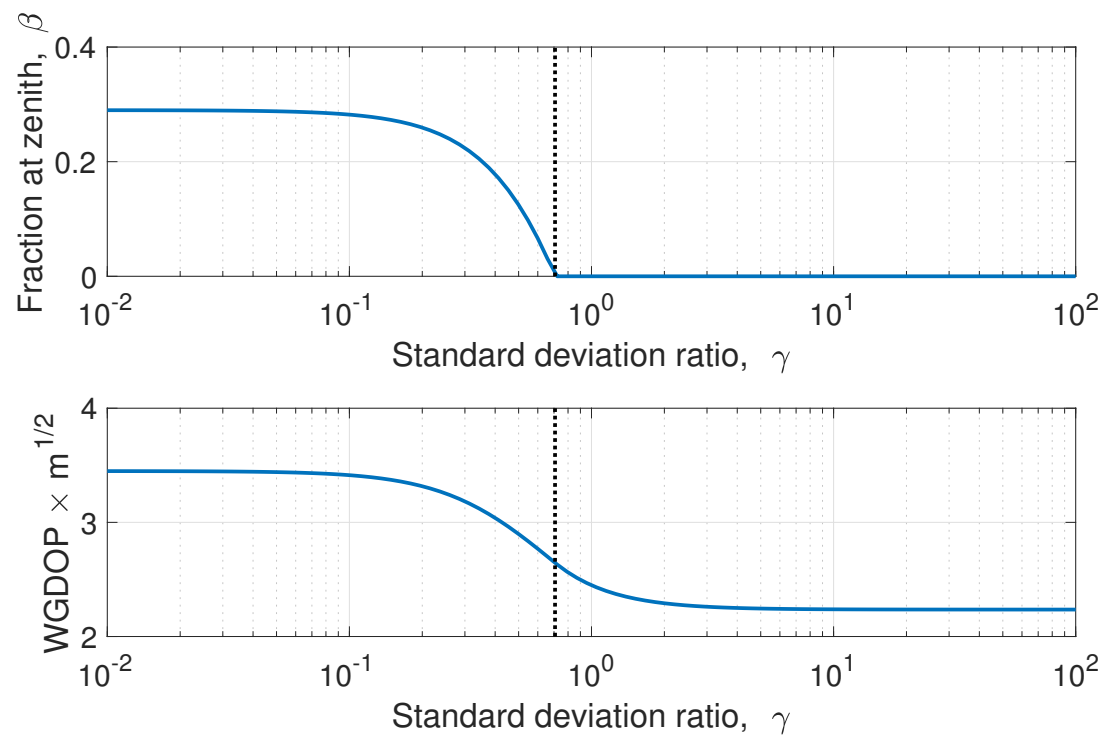

Figure 1: Results for a ranging source at nadir/zenith versus standard deviation ratio $\gamma$ : (top) the fraction of satellites at zenith, $\beta$, and (bottom) the coefficient in the expression for the WGDOP, $\sqrt{m} \times$ WGDOP .

- The bottom subplot shows the corresponding WGDOP performance; specifically, the coefficient of $\frac{1}{\sqrt{m}}$. For tiny $\gamma$ this coefficient approaches 3.45 (equivalent to GNSS alone), decreases with growing $\gamma$, and experiences diminishing returns once $\gamma^{2}$ exceeds one-half. Considering large $\gamma$, a very high quality ranging sensor at zenith reduces the WGDOP from $\frac{3.45}{\sqrt{m}}$ to $\sqrt{\frac{5}{m}}=\frac{2.24}{\sqrt{m}}$, a drop of about one-third.

One value of this theoretical analysis is that it provides a way to quantify the impact on GDOP of a high quality altimeter in aircraft applications. To validate the analysis, Figure 2 compares theory to actual computation for the case of $m=20$ satellites for selected variance ratios. For each circle, the number of satellites is rounded to the
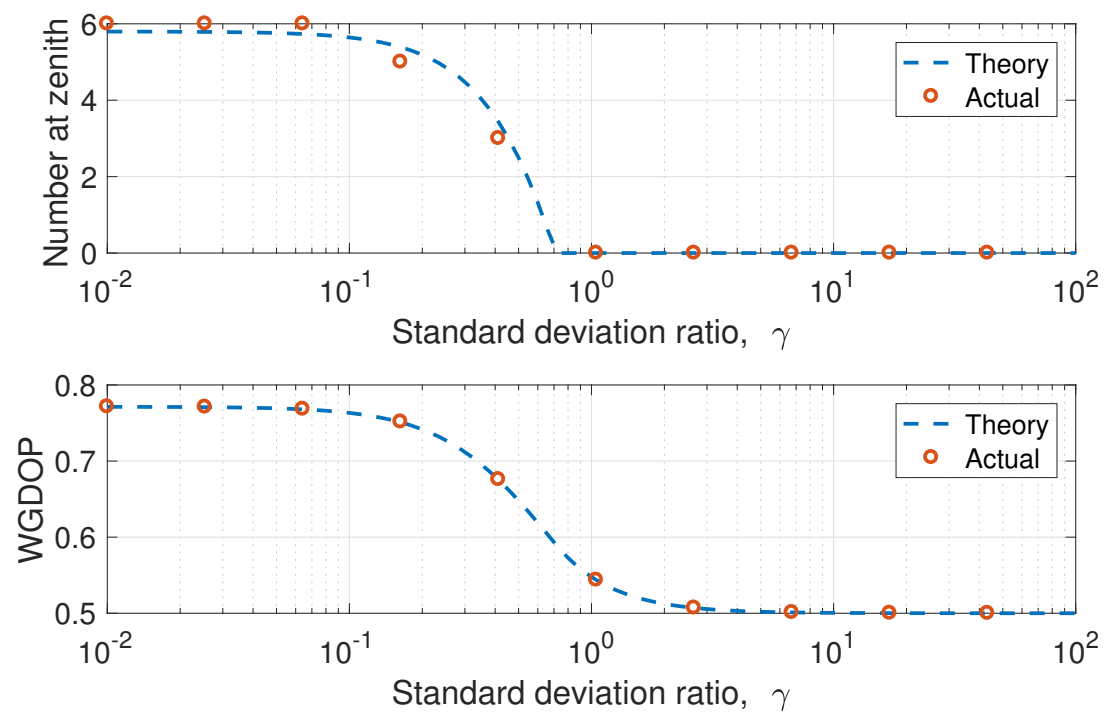

Figure 2: Performance versus standard deviation ratio $\gamma$ for $m=20$ total satellites: (top) the count of satellites at zenith (rounded to the best integer) and (bottom) the resulting WGDOP. 
nearest integer. The WGDOP is effectively on top of the bound.

From these curves we see that the most bang for the buck is achieved if $\gamma$ is near unity

$$
\gamma=\frac{\sigma}{\sqrt{m} \sigma_{r}}=1 \quad \text { or } \quad \sigma_{r}=\frac{\sigma}{\sqrt{m}}
$$

If the GNSS pseudorange accuracy is $\sigma=1$ meter and we have 9-12 satellites then to be useful the altimeter should have accuracy of $\sigma_{r} \approx 0.3$ meters ( 1 foot). An altimeter with $\sigma_{r}=10$ feet is essentially useless $(\gamma=0.1$ ).

\section{A Range to the Horizon}

As a second example, imagine that the ranging source is at the horizon relative to the receiver; without loss of generality, assume that the direction to the source is due north so that $e_{0}=0, n_{0}=1$, and $u_{0}=0$. With this restriction the matrix $\mathbf{G}^{T} \boldsymbol{\Gamma}^{-1} \mathbf{G}$ is

$$
\mathbf{G}^{T} \boldsymbol{\Gamma}^{-1} \mathbf{G}=\left[\begin{array}{cccc}
\sum_{k=1}^{m} e_{k}^{2} & \sum_{k=1}^{m} e_{k} n_{k} & \sum_{k=1}^{m} e_{k} u_{k} & \sum_{k=1}^{m} e_{k} \\
\sum_{k=1}^{m} e_{k} n_{k} & \frac{\sigma^{2}}{\sigma_{r}^{2}}+\sum_{k=1}^{m} n_{k}^{2} & \sum_{k=1}^{m} n_{k} u_{k} & \sum_{k=1}^{m} n_{k} \\
\sum_{k=1}^{m} e_{k} u_{k} & \sum_{k=1}^{m} n_{k} u_{k} & \sum_{k=1}^{m} u_{k}^{2} & \sum_{k=1}^{m} u_{k} \\
\sum_{k=1}^{m} e_{k} & \sum_{k=1}^{m} n_{k} & \sum_{k=1}^{m} u_{k} & m
\end{array}\right]
$$

and the optimization is as follows:

1. Set the red, off-diagonal terms to zero - this is equivalent to Eq. (1) from the nadir/zenith example.

2. Set the first two diagonal terms (in blue) equal to one another

$$
\sum_{k=1}^{m} e_{k}^{2}=\frac{\sigma^{2}}{\sigma_{r}^{2}}+\sum_{k=1}^{m} n_{k}^{2}=m \gamma^{2}+\sum_{k=1}^{m} n_{k}^{2}
$$

For smaller $\gamma$ this is possible, but as $\gamma$ grows it clearly becomes problematic. We discuss it further below.

3. Restrict the satellites to zenith or horizon, letting $\beta$ represent the number at zenith. In this case, using $n_{k}^{2}=1-e_{k}^{2}$ for horizon satellites, the condition of Eq. (2) can be written as

$$
\sum_{k=1}^{(1-\beta) m} e_{k}^{2}=\frac{m}{2}\left(\gamma^{2}+1-\beta\right)
$$

Since each $e_{k}^{2}$ is at most equal to one, the left hand side has maximum value of $(1-\beta) m$, so the condition cannot be satisfied if

$$
m \gamma^{2}>(1-\beta) m
$$

For example, let $(1-\beta) m=8$ (multiples of 4 provide simple examples to work with for this situation) and consider several values for $m \gamma^{2}\left(0.1,1,4\right.$, and 7.8) and their corresponding needed $\sum_{k=1}^{8} e_{k}^{2}$ values $(4.05,4.5$, 6 , and 7.9, respectively). The four subfigures in Figure 3 show sample satellite configurations (the horizon locations only, other configurations are possible) that would satisfy the balance equations for these four cases, respectively. For a very poor range sensor, the first case, the satellite locations are effectively equally distributed 

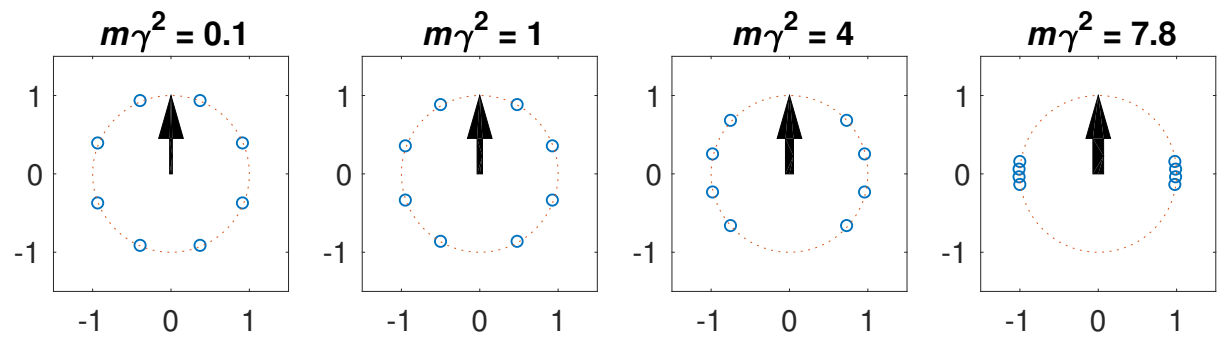

Figure 3: Horizon locations to satisfy balance for different quality ratios.

about the horizon (spacing of almost $45^{\circ}$ ). For an improving range sensor to the north (marked by the arrow), the horizon satellites maintain balance, but tend to be biased in the east-west direction (more generally, perpendicular to the direction to the range sensor). Finally, as $m \gamma^{2}$ approaches $(1-\beta) m$ the satellites converge to due east and west locations.

Assuming that we can meet the balance conditions, that $m \gamma^{2} \leq(1-\beta) m$, then the WGDOP bound is

$$
\text { WGDOP }\left.\right|_{\text {horizon }} \geq \sqrt{\left(\frac{4}{\gamma^{2}+1-\beta}+\frac{1+\beta}{\beta(1-\beta)}\right) \frac{1}{m}}
$$

If $m \gamma^{2}>(1-\beta) m$ so that the configuration on the horizon collapses to all satellites being east/west, the WGDOP bound is then

$$
\text { WGDOP }\left.\right|_{\text {horizon }} \geq \sqrt{\left(\frac{1}{1-\beta}+\frac{1}{\gamma^{2}}+\frac{1+\beta}{\beta(1-\beta)}\right) \frac{1}{m}}
$$

4. Optimizing the two expressions above yield several results:

- The dividing point in terms of $\gamma$ for the two solutions is $\gamma \approx \sqrt{\frac{3-\sqrt{3}}{2}} \approx 0.796$ (see Appendix B).

- For $\gamma>0.796$ the expression above is easily optimized, yielding $\beta=\frac{\sqrt{3}-1}{2} \approx 0.37$ for larger $\gamma$.

- For $\gamma<0.796$ the optimum choice for $\beta$ is the single positive root of

$$
5 \beta^{4}-2\left(\gamma^{2}+4\right) \beta^{3}+\gamma^{2}\left(\gamma^{2}-2\right) \beta^{2}+2\left(\gamma^{4}+3 \gamma^{2}+2\right) \beta-\left(\gamma^{4}+2 \gamma^{2}+1\right)=0
$$

(see Appendix B for details).

Figure 4 shows the results versus the sensor standard deviation ratio, $\gamma$ :

- The top subplot shows that fraction of satellites that should be at zenith, $\beta$. As expected, for vanishingly small $\gamma$ this ratio approaches $29 \%$, the fraction found in [9] for GNSS alone. As $\gamma$ increases, so does $\beta$ until $\gamma \approx 0.796$ for which the ranging sensor obviates the need for satellites in the same direction as the ranging source and all $m$ should be perpendicular to that direction (either directly overhead or east-west).

- The bottom subplot shows the corresponding WGDOP performance; specifically, the coefficient of $\frac{1}{\sqrt{m}}$. For tiny $\gamma$ this again approaches 3.45, decreases with growing $\gamma$, and experiences diminishing returns once $\gamma$ exceeds 0.796. A good quality ranging sensor at the horizon reduces the GDOP from $\frac{3.45}{\sqrt{m}}$ to $\frac{2.73}{\sqrt{m}}$, not quite as much of a reduction as was observed with a zenith/nadir ranging sensor (shown as the red dashed line).

As an example, Figure 5 compares theory to actual computation for the case of $m=20$ satellites for selected standard deviation ratios. For each circle, the number of satellites is rounded to the nearest integer. Again we see the step-like characteristic in the top subplot (and making even jumps due to the required symmetry - we could get examples with odd numbers of satellites at zenith by putting a satellite due south on the horizon and then balancing the northern components of the others), the WGDOP is effectively on top of the bound. 

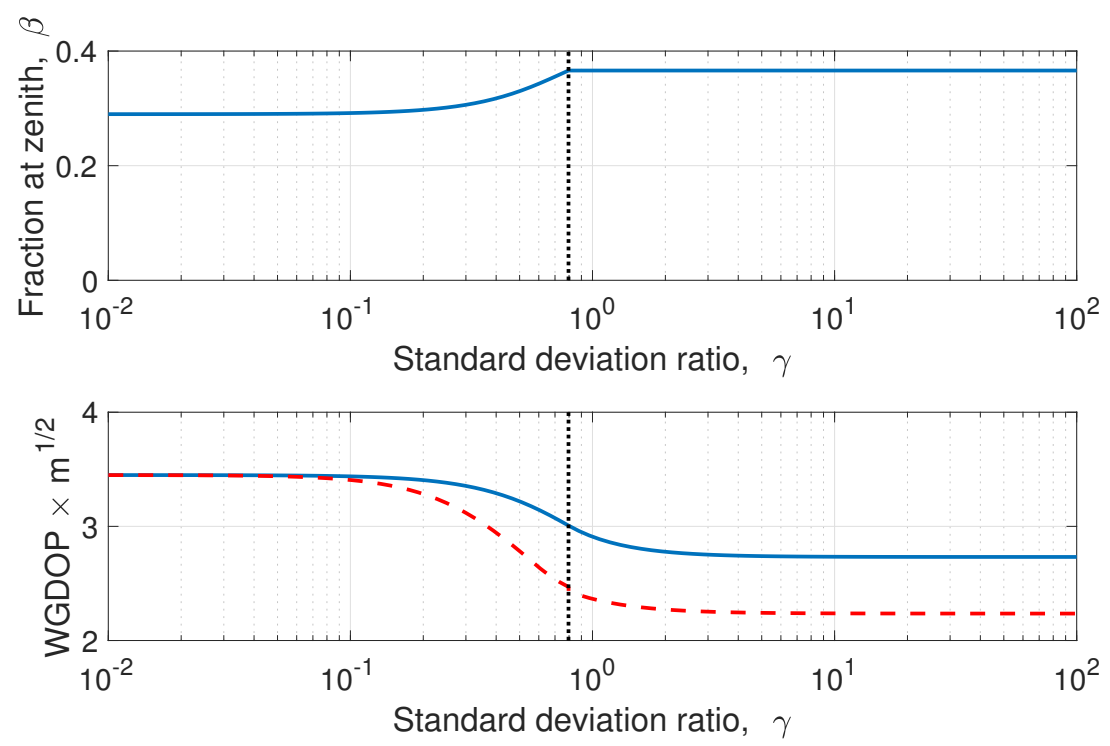

Figure 4: Results for a ranging source at the horizon versus variance ratio $\gamma$ : (top) the fraction of satellites at zenith, $\beta$, and (bottom) the coefficient in the expression for the WGDOP, $\sqrt{m} \times$ WGDOP. The red dashed line is the performance of a range to zenith/nadir.
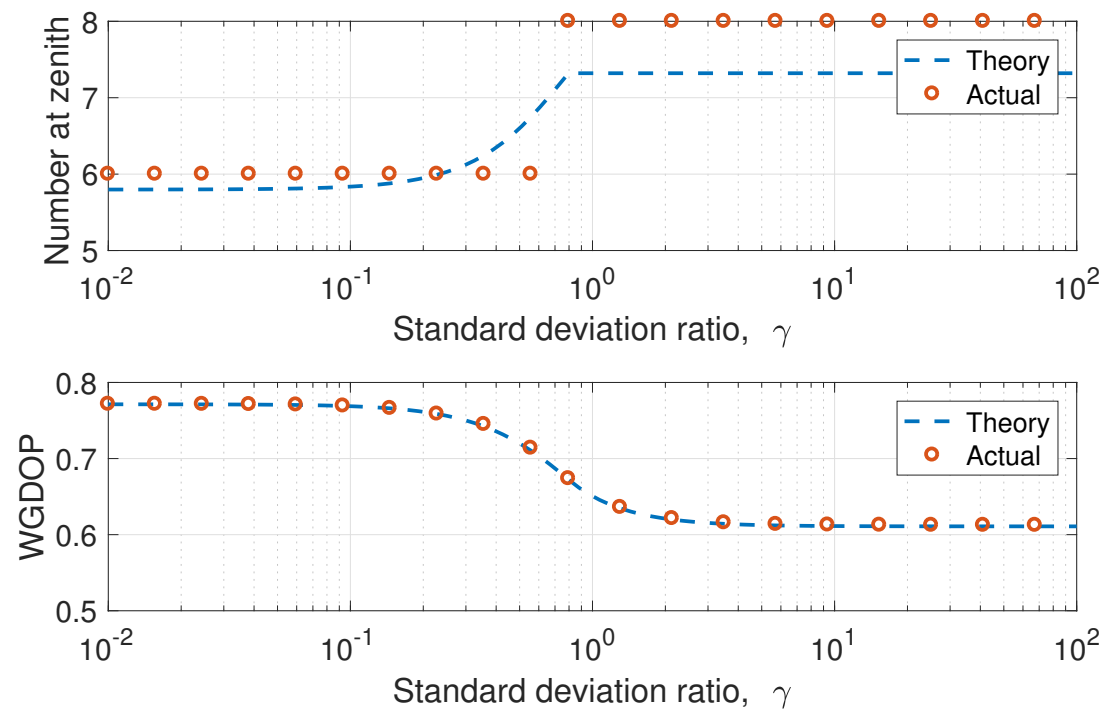

Figure 5: Performance versus variance ratio $\gamma$ for $m=20$ total satellites: (top) the count of satellites at zenith (rounded to the best integer) and (bottom) the resulting WGDOP.

\section{The General Case - A Slant Range}

Without loss of generality, let's assume that the ranging source is in the northerly direction (i.e. set $e_{0}=0$ ) and parameterize its unit vector by elevation angle $\theta$ so that

$$
n_{0}=\cos \theta \quad u_{0}=\sin \theta
$$


(i.e. negative $\theta$ for a ranging source below the receiver as in DME; an altimeter would have $\theta=-90^{\circ}$ ). The $\mathbf{G}^{T} \boldsymbol{\Gamma}^{-1} \mathbf{G}$ matrix is

$$
\mathbf{G}^{T} \boldsymbol{\Gamma}^{-1} \mathbf{G}=\left[\begin{array}{cccc}
\sum_{k=1}^{m} e_{k}^{2} & \sum_{k=1}^{m} e_{k} n_{k} & \sum_{k=1}^{m} e_{k} u_{k} & \sum_{k=1}^{m} e_{k} \\
\sum_{k=1}^{m} e_{k} n_{k} & \gamma^{2} m \cos ^{2} \theta+\sum_{k=1}^{m} n_{k}^{2} & \gamma^{2} m \sin \theta \cos \theta+\sum_{k=1}^{m} n_{k} u_{k} & \sum_{k=1}^{m} n_{k} \\
\sum_{k=1}^{m} e_{k} u_{k} & \gamma^{2} m \sin \theta \cos \theta+\sum_{k=1}^{m} n_{k} u_{k} & \gamma^{2} m \sin ^{2} \theta+\sum_{k=1}^{m} u_{k}^{2} & \sum_{k=1}^{m} u_{k} \\
\sum_{k=1}^{m} e_{k} & \sum_{k=1}^{m} n_{k} & \sum_{k=1}^{m} u_{k} & m
\end{array}\right]
$$

Immediately we see problems with invoking the 4 step optimization above; specifically, the red off-diagonal term (second row, third column)

$$
\gamma^{2} m \sin \theta \cos \theta+\sum_{k=1}^{m} n_{k} u_{k}
$$

cannot be made equal to zero if the satellites are limited to zenith and horizon. Clearly some modifications are needed. Toward this end, below we consider a simple constellation which is described by four parameters (and is optimum for the cases of GNSS alone, range to nadir/zenith, and range to the horizon), optimize its performance for the given slant angle $\theta$ and standard deviation ratio $\gamma$, and conjecture that its best performance is an achievable "bound".

Consider Figure 6. The left subfigure shows an optimum GNSS-only configuration [5]:

- Some fraction, $\beta m$, of the satellites are at zenith, $\left(e_{k}, n_{k}, u_{k}\right)=(0,0,1)$; these are represented by the blue square in this conceptual diagram.

- The remaining $(1-\beta) m$ satellites are on the horizon evenly spaced with $120^{\circ}$ separation. Each location consists of $\frac{(1-\beta) m}{3}$ satellites and they appear as the red circles.

In contrast, the right subfigure shows the situation (possibly exaggerated) with a slant range to the north and down (the black line representing the range direction). To balance the configuration the satellite locations have been perturbed:
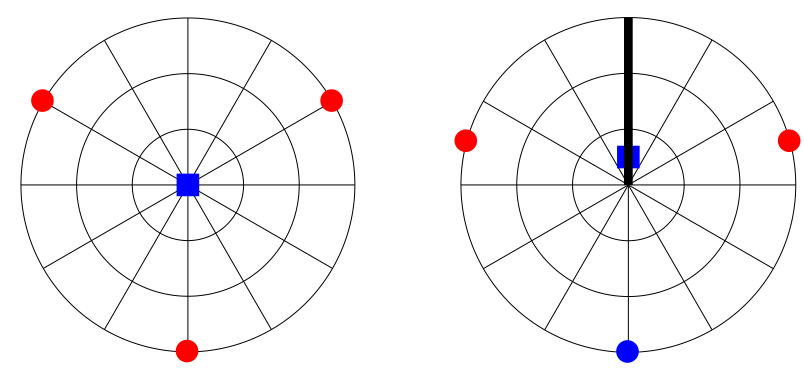

Figure 6: Conjectured slant range satellite configuration. 
- The satellites near zenith have been tilted northward to location $\left(e_{k}, n_{k}, u_{k}\right)=(0, \cos \phi, \sin \phi)$ for some elevation angle $\phi$. If the range direction was up, we would tilt these satellites toward the south.

- Some of the horizon satellites are rotated toward the south to locations, $\left( \pm \sin \psi, \cos \psi_{1}, 0\right)$; the others remain at azimuth $180^{\circ},\left(e_{k}, n_{k}, u_{k}\right)=(0,-1,0)$.

- We use different colors to indicate that there could be different numbers of satellites at these locations, $\beta m$ near zenith, $\delta(1-\beta) m$ on the horizon at azimuth $180^{\circ}$, and $(1-\delta)(1-\beta) m / 2$ each on the horizon toward the east and west.

For this perturbed constellation we have

$\mathbf{G}^{T} \boldsymbol{\Gamma}^{-1} \mathbf{G}=\left[\begin{array}{cccc}(1-\delta)(1-\beta) m \sin ^{2} \psi & 0 & 0 & 0 \\ & & \beta m \cos \phi \\ 0 & \gamma^{2} m \cos ^{2} \theta+\delta(1-\beta) m & \gamma^{2} m \sin \theta \cos \theta & +(1-\delta)(1-\beta) m \cos \psi \\ & +(1-\delta)(1-\beta) m \cos ^{2} \psi & +\beta m \sin \phi \cos \phi & -\delta(1-\beta) m \\ 0 & \gamma^{2} m \sin \theta \cos \theta & \gamma^{2} m \sin ^{2} \theta & \\ & +\beta m \sin \phi \cos \phi & +\beta m \sin ^{2} \phi & \\ 0 & \beta m \cos \phi & & \end{array}\right]$

Numerical optimization over the choice of $\beta, \delta, \phi$, and $\psi$ is quite direct. Figure 7 shows the resulting WGDOP for selected slant angles. We note that a range to zenith/nadir yields the most improvement while a range to the horizon provides the least. Further, the performance at $\theta$ and $-\theta$ are identical. As an example, Figure 8 shows the resulting parameter values for the case of $\theta=-15^{\circ}$ for $\gamma$ ranging from 0.01 to 100 :

- The top subfigure shows $\beta$ increasing from 0.29 for a poor sensor (effectively GNSS alone) and increasing to 0.35 as the sensor becomes excellent; this characteristic directly mimics what we saw for the horizon case in Figure 4 (although converging to a different fraction).

- The top subfigure also shows $\delta$ starting at one-third for a poor sensor and decreasing to zero as the sensor becomes excellent; this also mimics what we saw for the horizon case as the horizon satellites converged to due east and west.

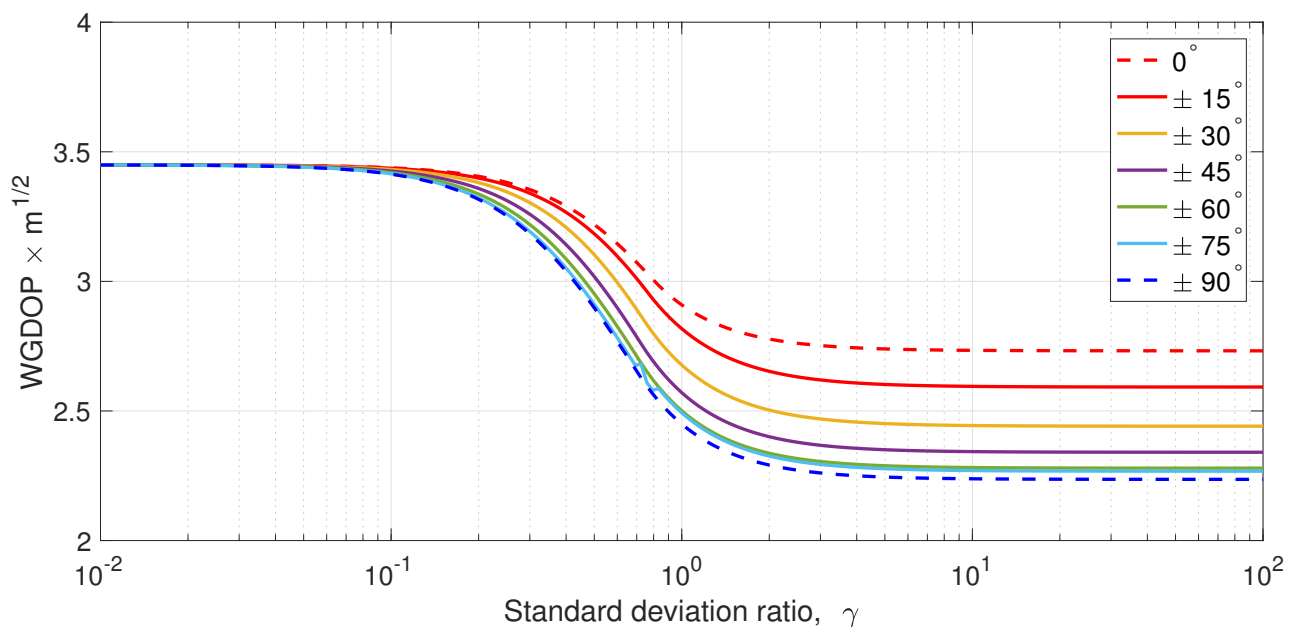

Figure 7: Conjectured WGDOP lower bounds for different slant angles. The extremes of the provable lower bounds for zenith/nadir and horizon are shown as dashed lines. 

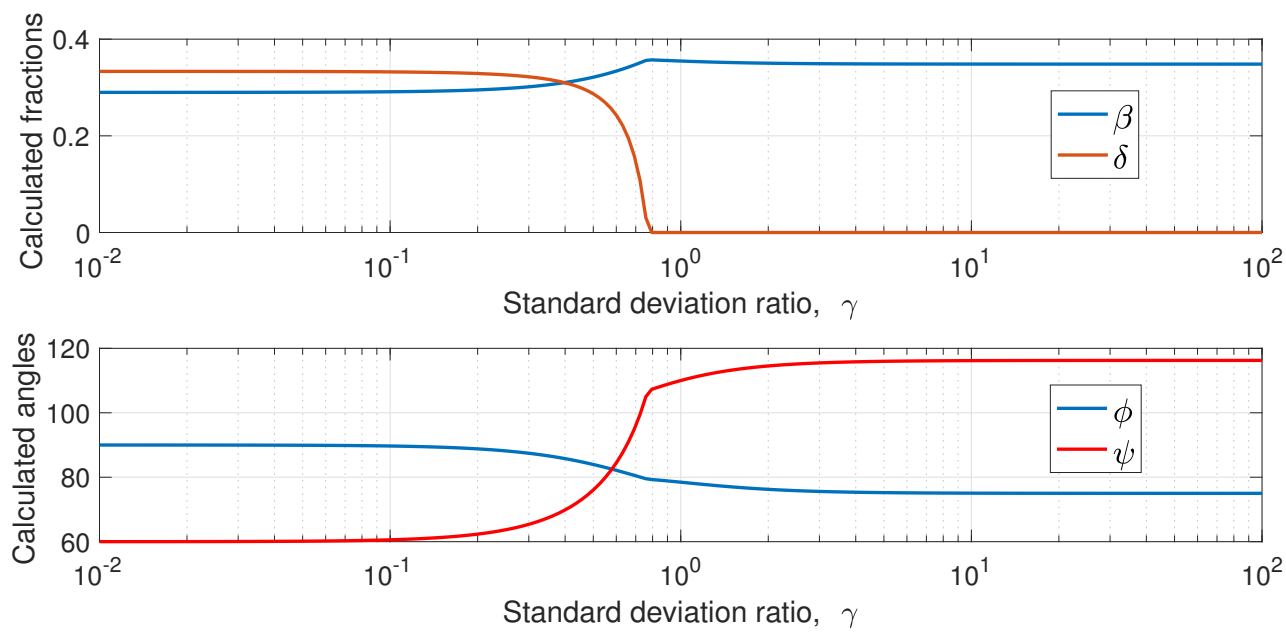

Figure 8: Parameters of the constellation at slant angle $\theta=-15^{\circ}$.

- The bottom subfigure shows that $\phi$, the zenith tilt, starts at $90^{\circ}$ and converges to $90+\theta$ as $\gamma \rightarrow \infty$. This convergence is expected as the constellation locates satellites in directions orthogonal to the range sensor.

- Finally, the bottom subfigure also shows that $\psi$, the east/west horizon locations, starts at $\pm 60^{\circ}$ and increases with improving range sensor. To date we have been unable to complete an asymptotic analysis (in $\gamma$ to understand this increase.

\section{Conclusions/Future}

We have developed a tight (i.e. achievable) lower bound to GDOP for the integration of a vertical (e.g. altimeter) or horizontal range measurement into a GNSS position solution. For a general slant range, we conjecture a bound based on a possible constellation.

Future work could include multiple (i.e. two) ranges and/or bearing measurements.

\section{Appendix A}

For the zenith/nadir situation, the question to resolve is the number of positive real roots of the polynomial

$$
5 \beta^{4}+(2 \gamma-8) \beta^{3}-12 \gamma \beta^{2}+(10 \gamma+4) \beta+4 \gamma^{2}-1=0
$$

Toward this end we invoke Sturm's theorem: 
Let $g(x)$ be a $n^{\text {th }}$ degree polynomial whose roots you are interested in. Define the finite sequence of polynomials

$$
\begin{aligned}
p_{0}(x) & =g(x) \\
p_{1}(x) & =\frac{d g(x)}{d x} \\
p_{2}(x) & =-\operatorname{rem}\left(\frac{p_{0}(x)}{p_{1}(x)}\right) \\
p_{3}(x) & =-\operatorname{rem}\left(\frac{p_{1}(x)}{p_{2}(x)}\right) \\
\vdots & \\
p_{n}(x) & =-\operatorname{rem}\left(\frac{p_{n-2}(x)}{p_{n-1}(x)}\right)
\end{aligned}
$$

in which $\operatorname{rem}(\cdot)$ is the remainder from the polynomial division (note that since we start with an $n^{\text {th }}$-order polynomial, then $p_{n}(x)$ is a constant). The count of real roots on an open interval $(a, b)$ of $x$ is the difference in the number of sign changes in the sequence of polynomials at the endpoints of the interval.

For the polynomial of interest, the sequence of polynomials (in the variable $\beta$ ) is

$$
\begin{aligned}
& p_{0}(\beta)=5 \beta^{4}+(2 \gamma-8) \beta^{3}-12 \gamma \beta^{2}+(10 \gamma+4) \beta+4 \gamma^{2}-1 \\
& p_{1}(\beta)=20 \beta^{3}+(6 \gamma-24) \beta^{2}-24 \gamma \beta+10 \gamma+4 \\
& p_{2}(\beta)=-\operatorname{rem}\left(\frac{p_{0}(f)}{p_{1}(f)}\right)=\ldots \\
& p_{3}(\beta)=-\operatorname{rem}\left(\frac{p_{1}(f)}{p_{2}(f)}\right)=\ldots \\
& p_{4}(\beta)=-\operatorname{rem}\left(\frac{p_{2}(f)}{p_{3}(f)}\right)=K, \text { a constant }
\end{aligned}
$$

where we have not supplied the details as the expressions are long, and not too insightful. Note that the constant $K$ is zero for $\gamma=0$, otherwise negative for $\gamma>0$.

Evaluating these polynomials at $\beta=0$ yields

$$
\begin{aligned}
& p_{0}(0)=4 \gamma^{2}-1 \\
& p_{1}(0)=10 \gamma+4, \text { positive for all } \gamma>0 \\
& p_{2}(0)=0.6-0.9 \gamma-3.75 \gamma^{2} \\
& p_{3}(0)=\ldots, \text { negative for all } \gamma>0 \\
& p_{4}(0)=K, \text { negative for all } \gamma>0
\end{aligned}
$$

Notice that irregardless of the actual sign of $p_{2}(0)$, this sequence has one or two sign changes, one if $p_{0}(0)$ is positive and two if $p_{0}(0)$ is negative. Similarly, evaluating at $\beta=1$ yields

$$
\begin{aligned}
& p_{0}(1)=4 \gamma^{2} \text { positive for all } \gamma>0 \\
& p_{1}(1)=-8 \gamma, \text { negative for all } \gamma>0 \\
& p_{2}(1)=-0.6 \gamma(7 \gamma+2), \text { negative for all } \gamma>0 \\
& p_{3}(1)=\ldots, \text { negative for all } \gamma>0 \\
& p_{4}(1)=K, \text { negative for all } \gamma>0
\end{aligned}
$$

The result of taking the difference of these counts is that the polynomial of interest has exactly one positive real root in the range $(0,1)$ if $p_{0}(0)$ is negative, or $\gamma<\frac{1}{2}$, and has no positive real root (i.e. we use $\beta=0$ as the minimizer) if $\gamma>\frac{1}{2}$. 


\section{Appendix B}

This appendix examines that case of a ranging source on the horizon (assumed, without loss of generality, to be due north); specifically, the optimization over $\beta$ for smaller values of $\gamma$.

The relevant WGDOP bound is

$$
\text { WGDOP }\left.^{2}\right|_{\text {horizon }} \geq\left(\frac{4}{\gamma^{2}+1-\beta}+\frac{1+\beta}{\beta(1-\beta)}\right) \frac{1}{m} \equiv \frac{g_{1}(\beta)+g_{2}(\beta)}{m}
$$

which we desire to minimize over the choice of $\beta$. Ignoring the $\frac{1}{m}$ term, we decompose the bound into two functions, $g_{1}$ and $g_{2}$, to allow for further examination:

- First, recall that the defined range for $\beta$ is $[0,1]$; the necessary condition for achievability restricts $\beta$ to the range $\left[0,1-\gamma^{2}\right]$. Further, this effectively limits our attention to variance ratio $\gamma \leq 1$.

- For $0 \leq \beta \leq 1$ the first component, $g_{1}(\beta)$, is positive, monotonically increasing, and convex as all three of the following expressions are positive for $\gamma>0$ :

$$
\begin{aligned}
& g_{1}(\beta)=\frac{4}{\gamma^{2}+1-\beta}>0 \\
& \frac{\partial g_{1}(\beta)}{\partial \beta}=\frac{4}{\left(\gamma^{2}+1-\beta\right)^{2}}>0 \\
& \frac{\partial^{2} g_{1}(\beta)}{\partial \beta^{2}}=\frac{8}{\left(\gamma^{2}+1-\beta\right)^{3}}>0
\end{aligned}
$$

- The second component, $g_{2}(\beta)$, requires a bit more examination. Looking at the function and its derivatives

$$
\begin{aligned}
& g_{2}(\beta)=\frac{1+\beta}{\beta(1-\beta)} \\
& \frac{\partial g_{2}(\beta)}{\partial \beta}=\frac{\beta^{2}+2 \beta-1}{\beta^{2}(1-\beta)^{2}} \\
& \frac{\partial^{2} g_{2}(\beta)}{\partial \beta^{2}}=2 \frac{\beta^{3}+3 \beta^{2}-3 \beta+1}{\beta^{3}(1-\beta)^{3}}
\end{aligned}
$$

there are several observations:

- The term itself is positive (not too surprising).

- Based upon the quadratic in its numerator of the second of these expressions (the denominator being positive), the slope of $g_{2}$ is clearly negative for $\beta<\sqrt{2}-1$ and positive for $\beta>\sqrt{2}-1$. Combining this with the fact that $g_{1}(\beta)$ is monotonically increasing, the obvious result is that the optimum choice for $\beta$ must fall in the range $[0, \sqrt{2}-1]$; hence, we would never want more than $41 \%$ of the satellites at zenth $(\sqrt{2}-1 \approx 0.41)$. Adding the necessary condition $\beta \leq 1-\gamma$, the optimum $\beta$ is restricted to the range $[0, \delta]$ with

$$
\delta \equiv \min \left\{\sqrt{2}-1,1-\gamma^{2}\right\}
$$

- The second derivative is positive for $0 \leq \beta \leq 1$ (the denominator being always positive, an application of Sturm's theorem to the numerator polynomial shows no roots in the interval) and the function is convex. 
- On the range $[0, \delta]$ the slope of $g_{1}$ is always positive and the slope of $g_{2}$ is always negative; hence, a minimum occurs whenever

$$
\frac{\partial g_{1}(\beta)}{\partial \beta}=-\frac{\partial g_{2}(\beta)}{\partial \beta}
$$

The equivalent condition on $\beta$ is that it is a root of the polynomial

$$
h(\beta) \equiv 5 \beta^{4}-2\left(\gamma^{2}+4\right) \beta^{3}+\gamma^{2}\left(\gamma^{2}-2\right) \beta^{2}+2\left(\gamma^{4}+3 \gamma^{2}+2\right) \beta-\left(\gamma^{4}+2 \gamma^{2}+1\right)=0
$$

- Since the sum of convex functions is also convex, if $g_{1}(\beta)+g_{2}(\beta)$ has a minimum at $\beta^{*}$ for $0 \leq \beta^{*} \leq \delta$ then the minimum is unique; if there is no point of zero slope on this range, then we use $\beta^{*}=\delta$ (with no point of zero slope the minimum occurs at one of the interval's endpoints, and since $g_{2}(\beta)$ is infinite at $\beta=0$ the minimum must occur at the right end point). The solution, then is

$$
\beta^{*}=\min \{\sqrt{2}-1,1-\gamma, \arg h(x)=0\}
$$

Some detailed analysis using Sturm's theorem (and well beyond the scope of this conference paper) shows that $h(x)$ has exactly one root on $(0,1)$ for $\gamma^{2} \leq \frac{3-\sqrt{3}}{2} \approx 0.634$ or $\gamma \leq \sqrt{\frac{3-\sqrt{3}}{2}} \approx 0.796$.

\section{References}

[1] P. Misra and P. Enge, Global Positioning System: Signals, Measurements and Performance, Massachusetts: Ganga-Jamuna Press, $2^{\text {nd }}$ ed., 2006.

[2] D. Gerbeth, M. Felux, M-S. Circiu, and M. Caamano, "Optimized selection of satellites subsets for a multiconstellation GBAS," Proc. ION Int'l. Tech. Mtg., Monterey CA, Jan. 2016.

[3] T. Walter, J. Blanch, and V. Kropp, "Satellite selection for multi-constellation SBAS," Proc. ION GNSS+ 2016, Portland OR, Sept. 2016.

[4] M. Zhang and J. Zhang, "A fast satellite selection algorithm: beyond four satellites," IEEE Jour. Sel. Topics Signal Proc., vol. 3, no. 5, Oct. 2009, pp. 740-747.

[5] P. F. Swaszek, R. J. Hartnett, and K. C. Seals, "Multi-constellation GNSS: new bounds on GDOP and a related satellite selection process," Proc. ION GNSS+ 2016, Portland OR, Sept. 2016.

[6] M. Kihara and T. Okada, "A satellite selection method and accuracy for the Global Positioning System," Jour. Navigation, vol. 31, no. 1, Spring 1984, pp. 8-20.

[7] J. J. Spilker, "Satellite constellation and geometric dilution of precision," Global Positioning System: Theory and Applications Volume 1, 1996, pp. 177-208.

[8] Y. Teng, J. Wang, and Q. Huang, "Mathematical minimum of geometric dilution of precision (GDOP) for dualGNSS constellations," Adv. Space Research, vol. 57, pp. 183-188.

[9] P. F. Swaszek, R. J. Hartnett, and K. C. Seals, "Lower bounds on DOP," Jour. Navigation, vol. 70, no.5, June 2017, pp.1041-1061. 